4 Crosby ET. Epidural catheter migration during labour: a hypothesis for inadequate analgesia. Can J Anaesth 1990; 37: 789-93.

5 Phillips $D C$, Macdonald $R$. Epidural catheter migration during labour. Anaesthesia 1987; 42: 661-3.

\section{Safety of alfentanil}

To the Editor:

Mulroy et al. ${ }^{1}$ and their publication entitled "Safety and efficacy of alfentanil and halothane in paediatric surgical patients" have declared that alfentanil is a safe anaesthetic, whether combined with nitrous oxide alone or with nitrous oxide and halothane. They have not specified in their methods their measure of safety nor have they identified the threshold used for declaration of safety of an anaesthetic agent.

The acceptability of an anaesthetic relates not only to the severity of minor adverse events associated with its use, but also to the frequency of major adverse events, such as anaphylaxis, late respiratory arrest, and severe metabolic derangement. I would contend that the safety of an anaesthetic agent is more frequently viewed in relation to the incidence of severe adverse events. The number of patients monitored in the aforementioned investigation is insufficient to demonstrate that the frequency of severe adverse events associated with the use of alfentanil is acceptably low.

When used in a scientific publication, the term safety must be defined both in terms of the type of complication studied and the threshold of acceptable frequency for the specified complication. Alfentanil may be a "safe" anaesthetic; safety, however, was neither measured nor demonstrated in the study by Mulroy et al.

\section{G.V. Goresky MD FRCPC}

Department of Anaesthesia

Alberta Children's Hospital, Calgary

\section{REFERENCE}

1 Mulroy JJ Jr, Davis PJ, Rymer DB, Chaitoff KA. Safety and efficacy of alfentanil and halothane in paediatric surgical patients. Can J Anaesth 1991; 38: 445-9.

\section{REPLY}

Dr. Goresky raises a pertinent and valid point about the use of the term "safe" in evaluating a medical therapy. Defining safety is not a simple task. If the dictionary definition "free from harm" is the criterion applied, perhaps no medical therapy is safe. The definition of safety of any agent or procedure cannot be reduced to a single factor but is based on a multitude of hazards balanced as a riskbenefit judgement by the practitioner.

We would agree that the size of the study that we reported is too small to analyze the incidence of uncommon events such as anaphylaxis and severe metabolic derangements. Therefore, we performed no such analysis. The size of a study needed to examine this aspect of safety and provide a statistically acceptable result for such rare events is dependent on the incidence of the events and on the definition of an acceptable incidence of these complications. For example, we have never observed a case of anaphylaxis, late respiratory depression, or severe metabolic derangement attributable to the use of alfentanil suggesting that it is an infrequent occurrence in our population.

The contention that the safety of an anaesthetic agent can only be measured in relation to the incidence of severe adverse events may be modelled using anaesthetic-related mortality as the ultimate indicator of safety. Tiret reported $a I$ in 40,000 incidence of anaesthetic-related mortality in children.' One would have to study an enormous number of patients to confirm the null hypothesis that there were no statistically significant differences in mortality among anaesthetic techniques. The relative risk of anaesthetic-related mortality between two different anaesthetic techniques could only be estimated by performing a case control study after both techniques had been used enough to produce mortality.

In order to demonstrate conclusively no statistical difference in the incidence, rare events among different techniques would require patient numbers far in excess of those in our study. Our goals were more modest. We focused on one aspect of the safety question. We attempted only to compare haemodynamic effects (using clinically relevant measures of heart rate and blood pressure) of alfentanil with those observed using halothane, an anaesthetic agent frequently employed in paediatric practice. We found that alfentanil was as safe (free from harm) to the patients as halothane was in the period studied.

The information that alfentanil in the doses studied may be safely administered to paediatric patients without producing haemodynamic depression greater than halothane is a small piece of the mosaic that defines the agent's safety and utility.

John J. Mulroy Jr. MD

University of Washington School of Medicine

Peter J. Davis MD

D. Ryan Cook MD

University of Pittsburgh School of Medicine

REFERENCE

1 Tiret L, Nivoche Y, Hatton F, Desmonts JM, Vourc'h G. Complications related to anaesthesia in infants and children. Br J Anaesth 1988; 61: 263-9.

\section{SIADH following minor surgery}

\section{To the Editor:}

Soroker $e t$ al. presented a case of the postoperative syndrome of inappropriate antidiuretic hormones (SIADH). ${ }^{1}$ We have had a similar case and others have been reported.

Our patient was a 33-yr-old woman with pelvic adhesions secondary to endometriosis. Laparotomy was scheduled and anaesthesia was induced with thiopentone and maintained with $\mathrm{O}_{2}: \mathrm{N}_{2} \mathrm{O}$, and isoflurane. Surgery was uneventful. Estimated blood loss was $150 \mathrm{ml}$, intravenous 
fluids were $2800 \mathrm{ml}$ of lactated Ringer's and normal saline. Urine output was $650 \mathrm{ml}$. In the post-anaesthesia care unit (PACU) she was awake with stable vital signs.

In the PACU the intravenous fluid was changed to $D_{5} \mathrm{~W}$. The next morning she was walking and taking ice chips. At 10:00 she complained of dizziness and a floating sensation; one hour later she complained of a headache. At 17:00 she was noted to be smiling and feeling drowsy but at 19:15 p.m. she was found unresponsive, cyanotic and pulseless. Cardiopulmonary resuscitation restored a haemodynamic function. Laboratory values were normal except for a plasma sodium concentration of 116 $\mathrm{mEq} \cdot \mathrm{L}^{-1}$, a chloride of $80 \mathrm{mEq} \cdot \mathrm{L}^{-1}$ and a glucose of 416 $\mathrm{mg} \cdot \mathrm{dl}^{-1}$. Computerized tomography showed diffuse cerebral oedema. A neurology consultation and an isoelectric EEG were obtained and the patient was declared brain dead. An autopsy showed hypoxic-ischaemic damage to the brain.

In 1986 Arieff reported 14 female patients with postoperative hyponatraemia who suffered grand mal seizures. ${ }^{2}$ These patients were of good health and underwent relatively minor procedures. Two patients had received only intravenous sedation, three had local anaesthesia and the others general anaesthesia. They were ambulatory, communicating, voiding spontaneously and taking fluids by mouth shortly after surgery. During the first two postoperative days the patients all complained of headache, nausea and vomiting. Later, half became incontinent of urine; four were described as hostile; and four were disoriented. Seven had hallucinations and eight were noted to be lying in bed with only mild symptoms just before having seizures. Serum sodium concentrations averaged 108 $\mathrm{mEq} \cdot \mathrm{L}^{-1}$ at that time. Within an hour of the seizures all suffered respiratory arrest. All were initially resuscitated but four died shortly thereafter and nine others remained in a persistent vegetative state. The other regained consciousness but suffered permanent partial paralysis.

Seven of Arieff's patients awoke after correction of their hyponatraemia. However, they all had neurological deterioration, suffered recurrent seizures and lapsed back into coma.

Soroker $e t$ al. presented a case of postoperative SIADH in a young boy. Previous cases have concerned only female patients thus postulating a sex influence. Perhaps these cases are not as rare as supposed but diagnosis and documentation occurs only rarely.

\footnotetext{
Thomas M. Fuhrman MD

Thomas Runyan MD

Thomas Reilley MD

Department of Anesthesiology,

Ohio State University Hospitals

Columbus, Ohio 43210-1228
}

\section{REFERENCES}

1 Soroker D, Ezri T, Feld S. Symptomatic hyponatremia due to inappropriate antidiuretic hormone secretion following minor surgery. Can J Anaesth 1991; 38: 225-6.

2 Arieff A. Hyponatremia, convulsions, respiratory arrest and permanent brain damage after elective surgery in healthy women. N Engl J Med 1986; 314: 1529-34.

\section{REPLY}

We have read with interest the case of postoperative hyponatraemia that ultimately progressed to coma and hypoxicischaemic damage to the brain, which was described by Fuhrman et al. in their letter.

The diagnosis of the syndrome of inappropriate antidiuretic hormone secretion (SIADH) should be confirmed by laboratory findings of urine osmolality hypertonic to serum and by demonstration of elevated serum ADH concentration, as not all postoperative hyponatraemia can be ascribed to the SIADH secretion. ${ }^{1,2}$ Therefore, the diagnosis of SIADH could not be definitively made in their case, or in the 15 cases described by Arieff. ${ }^{3}$

Furthermore, in our case the outcome was good, which is opposite to the grave outcome in the case desribed by Fuhrman et al. in their letter and in those of Arieff. ${ }^{3}$ Perhaps female sex influence predisposes to a less favourable outcome.

Some degree of inappropriate $A D H$ secretion is present in virtually all patients after surgery, ${ }^{\prime}$ and about $5 \%$ of postoperative patients have hyponatraemia (serum sodium $<130$ $\left.m E q \cdot L^{-1}\right) ;{ }^{\prime}$ however, the condition is rarely symptomatic. The purpose of our report ${ }^{4}$ was to describe a rare and well documented symptomatic occurrence of SIADH secretion after minor surgery.

Samuel Lurie MD

Tiberiu Ezri MD

Irena Savir MD

David Soroker MD

Department of Anesthesia

Kaplan Hospital

Rehovot, Israel

\section{REFERENCES}

1 Robertson GL, Shelton RI, Athar S. The osmoregulation of vasopressin. Kidney Int 1976; 10: 25-37.

2 Cung HM, Kluge R, Schrier RW, Anderson RJ. Postoperative hyponatremia: a prospective study. Arch Intern Med 1986; 146: 333-6.

3 Arieff AI. Hyponatremia, convulsions, respiratory arrest, and permanent brain damage after elective surgery in healthy women. N Engl J Med 1986; 314: 1529-34.

4 Soroker D, Ezri T, Lurie S et al. Symptomatic hyponatraemia due to inappropriate antidiuretic hormone secretion following minor surgery. Can J Anaesth 1991; 38: 225-6. 